\title{
MODELO DE CRECIMIENTO DIAMETRAL PARA ALGUNOS RENOVALES DE RAULI
}

\author{
Victor Cubillos D* \\ RESUMEN
}

Mediante un enfoque de análisis del árbol individual y evaluación de la competencia, se construyó un modelo de crecimiento en diámetro para individuos de raulí (Nothofagus alpina 'Poepp. et Endl.' Oerst.) y el cual considera las variables de estado de los árboles y de sus competidores directos. La información de crecimiento se obtuvo por el método de análisis del tallo. El modelo se construyó mediante un análisis de regresión y correlación múltiple.

El estudio se efectuó en renovales mixtos de raulí en el área de Panguipulli - Neltume, a $39^{\circ} 45^{\prime}$ de latitud Sur, comuna de Panguipulli, provincia de Valdivia, en la Décima Región de Chile.

Como resultado se presentan tres modelos generales, que incluyen variables de estado de los árboles y de la competencia. El modelo más preciso es el que considera como variables independientes el diámetro a la altura del pecho (DAP), el diámetro de copa de los árboles, la distancia media entre el árbol y los competidores, la edad, la altura de inicio de la copa de los árboles analizados y de su competencia.

El modelo seleccionado tiene una bondad de ajuste de 0,91 en términos de correlación $\mathrm{r}$ y un error cuadrático medio (ECM \%) de $23,7 \%$. Se efectuó además un análisis de sensibilidad, presentándose una tabla con los valores de incremento en diámetro más significativos.

\begin{abstract}
General information concerned with stocks and actual condition of young stands of Nothofagus alpina (Poepp, et endl) Oerst), "Rauli" are presented in this article. These aspects are tipified through stade variables both for the Rauli trees and for other surrounding species.

The study was undertaken in young Rauli stands located in the Panguipulli-Neltume area, $39^{\circ} 46^{\prime}$ ' south latitude, Province of Valdivia X Región, Chile.

Growth information was obtained by stem analysis. A model was constructed applain regression analysis and multiple correlation.

As a result, three general models are presented which include individual state variables for the individuals and sorrounding competitors. The best model includes variables such as: diameter bresast height (d.b.h.) crown diameter, age, medium distance to competitors, height to first limb.

The best model has a correlation of $r=0.91$. Also included is a sensitivity analysis for this model showing a table with values of diameter increments.
\end{abstract}

*Ingeniero Forestal, U. de Chile. División Regional, Instituto Forestal, Barros Arana 121. Concepción - Chile. 


\section{INTRODUCCION}

Para estimar el crecimiento futuro de los árboles se utilizan modelos matemático-estadísticos que predicen el crecimiento de los individuos en un período determinado.

En el caso del raulí, existen modelos de crecimiento diametral construidos a través de una relación lineal simple entre el crecimiento en diámetro y el diámetro a la altura del pecho con corteza (DAPcc) (SOLER, 1979; HERRERA, 1976). Aunque este tipo de modelos son de gran utilidad práctica, están limitados por el hecho de explicar el incremento en diámetro a través de una sola variable de estado, el DAP, con exclusión de otras que pueden contribuir a explicar en forma más completa el incremento en diámetro.

Como una forma de superar esa limitación, este estudio presenta un modelo de crecimiento diametral construido a partir de diversas variables de estado de árboles individuales de raulí sometidos a distintos regímenes de competencia, incorporando también la información referente a los individuos competidores (Grosse, Cubillos, Bourke, 1986).

El modelo de crecimiento permite proyectar el crecimiento de individuos jóvenes de raulí y sirve, en consecuencia, como elemento de apoyo para la planificación de intervenciones silvícolas aplicadas a renovales mixtos de esta especie, con el fin de incrementar su rendimiento.

\section{ANTECEDENTES GENERALES}

Los renovales considerados en el estudio son bosques de segundo crecimiento que tuvieron su origen en talas rasas seguidas por roces a fuego y que presentan características de coetaneidad (CONAF, 1986). La edad de aproximadamente el 95\% de la superficie de los renovales estudiados se encuentra entre los 20 y los 50 años, siendo de mayor frecuencia el rango de edad entre los 20 y los 40 años. El diámetro medio general es de $30 \mathrm{~cm}$, el área basal fluctúa entre los $10 \mathrm{y} \operatorname{los} 60 \mathrm{~m}^{2} /$ ha y el número de árboles por hectárea varía entre 600 y 5000 (Puente, 1979).

Existencias de renovales mixtos. De acuerdo con información reciente, la superficie total de renovales coetáneos existentes en el país asciende a 300.000 ha, de las cuales el $85 \%$ se localiza en las Regiones VIII, IX y X (CONAF, 1986). Las especies de mayor valor comercial presentes en estos renovales son: Nothofagus alpina (raulí), Nothofagus obliqua (roble), Nothofagus dombeyi (coigüe), Nothofagus glauca (hualo), Laurelita phillipiana (tepa) y Saxegothea conspicua (mañío).

Caracteristicas de los renovales muestreados. El estudio se realizó en renovales del tipo forestal coigüe-rauli-tepa (Donoso, 1981), en el área de Panguipulli-Neltume en la Cordillera de Los Andes, a $39^{\circ} 46^{\prime}$ de latitud Sur, provincia de Valdivia, comuna de Panguipulli, Décima Región.

Las áreas específicas consideradas para el estudio se conocen como "Los Hornos", "Quebrada Honda" (sectores 1 y 2 ) y "Puerto Fuy". La conformación de los renovales de este sector, originados a partir de bosques naturales que se regeneraron por semillas o tocón, coincide con la definición general dada por Puente et al. (1979).

Para el análisis se consideraron rodales coetáneos entre los 25 y los 40 años. La presencia numérica está representada mayoritariamente por Nothofagus alpina (raulí), secundariamente por Nothofagus obliqua (roble) y como especies acompañantes Laurelia phillipiana (tepa) y Saxegothea conspicua (mañio).

Las situaciones que se muestrearian se seleccionaron considerando distintas etapas de desarrollo dentro de la fase de crecimiento en la que se encuentran los renovales. De acuerdo con este criterio, se seleccionan los sectores "Quebrada Honda" y "Puerto Fuy" para los renovales más jóvenes y "Los Hornos" para el análisis de rodales de mayor edad.

Los tres sectores seleccionacios fueron raleados hace aproximadamente diez años, como 
parte de un programa de manejo silvícola. La intervención realizada se puede definir como un raleo selectivo por lo alto y por lo bajo, puesto que el criterio aplicado fue dejar en pie sólo aquellos árboles que presentaban la mayor posibilidad potencial de acumular volumen aserrable durante el resto de la rotación. La intervención dejó como árboles seleccionados únicamente ejemplares de raulí y roble, especies entre las cuales la competencia resultó reducida.

\section{METODOLOGIA}

Definición del árbol sujeto y sus competidores. El modelo diseñado incluye dos tipos de variables, las del sujeto y las del competidor. Como variables del sujeto se entienden las variables de estado del árbol observado, al cual se le hizo análisis de tallo. Las variables del competidor corresponden al valor promedio de las variables de estado de los árboles cercanos al sujeto y que influyen en términos de competencia sobre éste. La selección de los árboles competidores se estableció básicamente por distancia: el entorno del árbol seleccionado se dividió en cuatro cuadrantes y se consideró como árbol competidor al más cercano en cada uno de ellos. Se obtuvieron asi cuatro competidores por cada árbol sujeto. La selección de los competidores tomó también en cuenta su proyección de copa sobre el árbol sujeto.

Obtención de la información básica. Para determinar el crecimiento histórico de los individuos se efectuó un análisis de tallo, midiendo los anillos de crecimiento en rodelas obtenidas por cortes transversal a distintas alturas del fuste. (PRODAN, 1965).

Las rodelas extraídas del árbol se secaron en laboratorio a $120^{\circ} \mathrm{C}$ durante 18 horas con aire forzado, hasta llegar a un contenido de humedad aproximado al $12 \%$. Luego se lijó una de las caras de la rodela para hacer más visibles los anillos de crecimiento anual. Posteriormente se midió el espesor y el número de anillos en cuatro radios de cada rodela y se registró la información para cada árbol independiente. Las mediciones se realizaron partiendo desde la corteza hacia la médula del árbol.

La información de crecimiento pasado así obtenida fue procesada mediante el programa computacional de análisis de tallo ANATAL (Alvarez, 1977).

Los resultados del proceso de la información son tablas de diámetro sin corteza (DAPsc), área basal, altura total y volumen cúbico en función de la edad, con sus respectivos crecimientos anuales medios (CAM) y periódicos (CAP).

Esta información permitió establecer una relación entre el incremento en diámetro registrado en los últimos cinco años y las variables de estado del árbol sujeto y sus competidores.

Estimación del incremento en diämetro. El incremento diametral anual promedio (IDAPA) se obtuvo promediando el crecimiento anual registrado en cada uno de los cinco últimos años por cada individuo de una muestra de 75 árboles de raulí a los cuales se les efectuó análisis de tallo.

Con la información de las variables del árbol sujeto y sus competidores, junto con el in-) cremento en diámetro promedio de los últimos cinco años (IDAPA), se construyó un archivo de trabajo básico. Con dichos datos se efectuaron análisis de regreción y comclación simple y mul. tiple, según el esquema de aplicación presentado en el diagrama 1.

El grado de dependencia entre el incremento diametral anual y las variables de estado se evaluó a través del coeficiente de correlación (r) y el error cuadrático medio (ECM\%).

En la Tabla 1 se presentan las variables de estado del árbol sujeto y sus competidores incluidas en los modelos de crecimiento: 


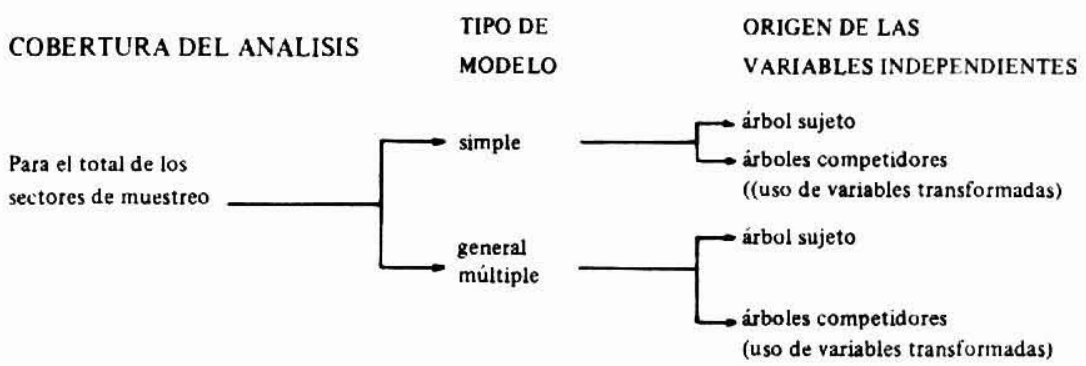

DIAGRAMA 1: Aplicación del análisis de regresión y correlación para el incremento diametral promedio anual (IDAPA) como variable dependiente.

\section{TABLA 1}

VARIABLES DE ESTADO MEDIDAS

\begin{tabular}{|c|c|}
\hline Clave & Explicación de la variable (unidad) \\
\hline ID $\backslash P A$ & incremento en diametro promedio anual $(\mathrm{cm}$ ) \\
\hline DC & diametro de copa $(\mathrm{m})$ \\
\hline DISTMCO & distancia media al competidor $(\mathrm{m})$ \\
\hline DAP & diametro a $1.3 \mathrm{~m}$ de altura $(\mathrm{cm})$ \\
\hline HT & altura total de lus arboles $(\mathrm{m})$ \\
\hline t & edad (añas) \\
\hline VT & volumen total $\left(\mathrm{m}^{3}\right)$ \\
\hline HIC & altura de inicio de la copa (m) \\
\hline IHA & incremento en altura anual $(\mathrm{m})$ \\
\hline IVOA & incremento en volumen anual $\left(\mathrm{m}^{3}\right.$, \\
\hline DCMCO & diametro medio de copa del competidor (m) \\
\hline DAPMCO & diametro medio del competidor medido a $13 \mathrm{~m}$ de altura (cm) \\
\hline HTCO & altura total del competidor $(\mathrm{m})$ \\
\hline HICCO & altura de incio de copa del competidor (m) \\
\hline EC & espacio de copa $-\mathrm{DC}^{2} \mathrm{x}(\mathrm{HT} \cdot \mathrm{HIC})\left(\mathrm{m}^{3}\right)$ \\
\hline$E C D$ & espacio de copa por la distancia $-\mathrm{DC}^{2} \times(\mathrm{HT} \cdot \mathrm{HIC}) \times$ DIST MCO \\
\hline & indice de competencia $(1)=\underline{D C}+\mathrm{DCMCO}$ \\
\hline 1001 & $2 \times$ DISTMCO \\
\hline \multirow{2}{*}{$\mathrm{HO}_{2}$} & \multirow{2}{*}{ indice de cumpetencia (2) $\frac{\text { DAP }}{\text { DAPMCO } \text { DISTMCO }}$} \\
\hline & \\
\hline $\ln$ & logantmo natural en base $e$ \\
\hline
\end{tabular}

\section{RESULTADOS}

Caracteristicas cuantitativas. En el área de estudio se caracterizan cuatro sectores con parcelas temporales de muestreo, en las cuales se midieron las variables de estado de todos los individuos. Los sectores de muestreo son: Los Hornos, Quebrada Honda sector 1, Quebrada Honda sector 2 y Puerto Fuy.

Las variables de estado raulí y del total de especies se presenta en el Cuadro 1 ordenadas por sector y parcela.

Estadistica descriptiva de las variables de estado y del incremento diametral de los árboles sujeto. La caracterización de las variables involucradas en la construcción del modelo de crecimiento permite conocer el comportamiento de la muestra en relación a su dispersión y valores medios. En el Cuadro 2 se muestran los valores medios, la varianza, la desviación estándar y el coeficiente de las variables analizadas. 


\section{CUADRO 1}

\section{VARIABLES DE ESTADO DE LA ESPECIE RAUL Y DEL TOTAL DE ESPECIES EN CADA PARCELA DE LOS DISTINTOS SECTORES EN ESTUDIO}

\begin{tabular}{|c|c|c|c|c|c|c|c|c|c|c|c|c|c|c|c|c|c|}
\hline \multirow{3}{*}{$\begin{array}{l}\text { Sector } \\
\text { Los Hor }\end{array}$} & \multirow[b]{2}{*}{ Parcela } & \multirow{2}{*}{\multicolumn{2}{|c|}{$\begin{array}{l}\text { No.de Arb. } \\
\text { por ha } \\
\text { Nha }\end{array}$}} & \multirow[b]{2}{*}{$\begin{array}{l}\text { DAP Medio } \\
\bar{D} \text { en cm }\end{array}$} & \multirow{2}{*}{\multicolumn{2}{|c|}{$\begin{array}{l}\text { Diam.Medio } \\
\text { cuadratico } \\
\text { DCLAD (cm) }\end{array}$}} & \multirow[b]{2}{*}{$\begin{array}{l}\text { Edad } \\
\text { en } \\
\text { Antos }\end{array}$} & \multirow[b]{2}{*}{$\begin{array}{l}\text { Area Basal } \\
\text { AB en m } m^{2} \mathrm{ha}\end{array}$} & \multirow{2}{*}{\multicolumn{2}{|c|}{$\begin{array}{l}\text { Altura Total } \\
\text { en } m \text {. }\end{array}$}} & \multirow{2}{*}{\multicolumn{2}{|c|}{$\begin{array}{l}\text { Cob. de las } \\
\text { Copas en } \\
\text { Porcentayes }\end{array}$}} & \multirow{2}{*}{\multicolumn{2}{|c|}{$\begin{array}{l}\text { Alt. de inic. } \\
\text { de la Copa } \\
\text { HINIC en m }\end{array}$}} & \multicolumn{3}{|c|}{ Incremento Anuales } \\
\hline & & & & & & & & & & & & & & & \multirow{2}{*}{$\begin{array}{c}\begin{array}{c}\text { Diám. } \\
\text { IDAPA } \\
\text { (cm) }\end{array} \\
0.24\end{array}$} & \multirow{2}{*}{$\begin{array}{l}\text { Nt. } \\
\text { IHA } \\
\text { (m) }\end{array}$} & \multirow{2}{*}{$\begin{array}{l}\begin{array}{l}\text { Vol. } \\
\text { IVOA } \\
\left(\mathrm{m}^{3}\right)\end{array} \\
9.008\end{array}$} \\
\hline & 1 & 1.344 & 1728 & 16991786 & 1861 & $200 ?$ & 36 & 36.5454 .641 & 1996 & 18.669 & $97: 5$ & 13465 & 10.56 & 906 & & & \\
\hline Los Hornos & 2 & $1: 200$ & 1536 & 17411761 & 1922 & 2009 & 36 & $3483+8.991$ & 16.17 & $1+09$ & 11963 & 150.01 & B. 20 & 642 & 0.37 & 032 & 0.049 \\
\hline Las Hornos & 3 & +32 & -84 & 23122229 & 2519 & 2447 & 42 & $\therefore 5336921$ & 1996 & 1785 & $6-21$ & 10198 & 896 & 81 & 056 & $0+1$ & 0025 \\
\hline Quebtada & 4 & 800 & 1280 & 12651500 & $13+4$ & 1836 & 26 & 113437.931 & 1198 & $10 \leq 1$ & $71: 6$ & 11156 & 516 & 355 & 081 & 039 & 0015 \\
\hline Hionda & 5 & -52 & 1056 & $12: 2013.03$ & 13.02 & 1480 & 25 & 100118831 & 1211 & 984 & $76-9$ & 10324 & +94 & 336 & 0.84 & 055 & 9012 \\
\hline Sevtor 1 & 6 & 464 & 768 & $13: 21303$ & $14: 1$ & 1411 & 24 & 28912.041 & $10: 3$ & 903 & $44+0$ & 6192 & +79 & $3+3$ & 095 & 042 & 0012 \\
\hline $\begin{array}{l}\text { Quebrada } \\
\text { Honda }\end{array}$ & 7 & 352 & 736 & 17.9119 .09 & 1907 & $24: 3$ & 26 & 100535331 & 1416 & 1261 & 5985 & 10261 & 6.81 & $4+6$ & 0.88 & $0+45$ & 0017 \\
\hline Sector 2 & 8 & 336 & 672 & $201917: 17$ & 2078 & 18.18 & 24 & 11.4017801 & 1551 & 1182 & 62.72 & 85.38 & 630 & +18 & 1.12 & $0+4$ & 0.023 \\
\hline \multirow[t]{2}{*}{ Puerto Fuy } & 9 & 416 & 944 & 122013.87 & 12.68 & 14.38 & 30 & 5.2515 .491 & 13.83 & 1449 & 3901 & 9796 & 617 & $6+0$ & $0.3 ?$ & $0+$ & 0005 \\
\hline & 10 & 352 & 944 & $1351 \quad 17.17$ & 15.23 & 22.74 & 31 & 6.4140 .831 & 13.95 & 15.17 & 50.20 & 14100 & 575 & 591 & 059 & 0.59 & 0010 \\
\hline
\end{tabular}

\section{CUADRO 2}

\section{ESTADISTICA DESCRIPTIVA DE LAS VARIABLES DE ESTADO Y DEL INCREMENTO DIAMETRAL DE LOS ARBOLES SUJETO (SE CONSIDERARON 75 ARBOLES)}

\begin{tabular}{lrrrr}
\hline Variable & Media & Varianza & Des. Están. & Coef. Var. \\
\hline DC & 3.8773 & 2.1923 & 1.4806 & 0.381874 \\
HIC & 6.7706 & 0.7047 & 3.1152 & 0.460110 \\
DAP & 17.0839 & 52.3250 & 7.2336 & 0.423416 \\
HT & 15.0479 & 18.3654 & 4.2855 & 0.284789 \\
E & 29.6133 & 68.5644 & 8.2804 & 0.279616 \\
IHA & 0.4357 & 0.0181 & 0.1347 & 0.309063 \\
IVOA & 0.0185 & 0.0014 & 0.0376 & 2.033661 \\
VT & 0.1785 & 0.0285 & 0.1687 & 0.945046 \\
DISTM & 3.6573 & 1.9781 & 1.4065 & 0.384562 \\
IDAPA & 0.6884 & 0.1469 & 0.3833 & 0.556740 \\
\hline
\end{tabular}

El incremento volumétrico (IVOA) presenta el coeficiente de variación más alto (203\%), seguido por el volumen total (95\%), lo que indica una cierta heterogeneidad del rodal, presumiblemente producida por la competencia entre los individuos.

Los bajos coeficientes de variación obtenidos para las variables edad $(28 \%$ o) y altura total $(29 \%$ o) pueden tomarse como indicadores de una cierta coetaneidad del rodal.

Matriz de correlación múltiple. La matriz de correlación múltiple es un arreglo de una submatriz inferior, donde las filas y columnas representan las variables del árbol sujeto y los incrementos en diámetro, altura y volumen promedio anual. Cada elemento de la matriz es el coeficiente de correlación entre las variables mencionadas. En la matriz de correlación múltiple es posible visualizar conjuntamente el grado de dependencia entre las distintas variables de estado de los árboles (Cuadro 3). 


\section{CUADRO 3}

\section{MATRIZ DE CORRELACION MULTIPLE ENTRE LAS VARIABLES DE ESTADO DEL ARBOL SUJETO Y LOS INCREMENTOS ANUALES PROMEDIO EN DIAMETRO, ALTURA Y VOLUMEN}

\begin{tabular}{lrrrrrrrrrr}
\hline & \multicolumn{1}{l}{ DC } & HIC & DAP & HT & E & IHA & IVOA & VT & DISTM & IDAPA \\
\hline DC & 1.00000 & & & & & & & & & \\
HIC & .08651 & 1.00000 & & & & & & & & \\
DAP & .83566 & .33840 & 1.00000 & & & & & & & \\
HT & .36576 & .69639 & .67774 & 1.00000 & & & & & & \\
E & .13672 & .60473 & .45272 & .82344 & 1.00000 & & & & & \\
IHA & .41285 & -.03776 & .25795 & .01158 & -.10693 & 1.00000 & & & & \\
IVOA & .37969 & .21650 & .43904 & .29525 & .24206 & -.06752 & 1.00000 & & & \\
VT & .68642 & .36460 & .89938 & .74716 & .61839 & .05855 & .46049 & 1.00000 & & \\
DISIM & .28953 & -.00828 & .26853 & -.01618 & -.04982 & .12367 & -.03981 & .18861 & 1.00000 \\
IDAPA & .71567 & -.14924 & .54525 & -.06946 & -.36823 & .52139 & .19593 & .23862 & .350751 .00000 \\
\hline
\end{tabular}

Las correlaciones más altas se obtuvieron entre el diámetro a la altura del pecho (DAP) y el volumen total (VT), con un 0,90 ; el DAP y el diámetro de copa (DC) con un 0,84 ; la edad (E) y la altura total (HT) con 0,82; el VT y la edad (E) con 0,62 y entre el incremento medio diamétrico (IDAPA) y el diámetro de copa (DC), con 0,71 .

Correlaciones obtenidas entre el incremento diametral anual y las variables del sujeto y sus competidores. A continuación se presentan las correlaciones obtenidas entre el incremento diamétrico (IDAPA) y las variables de estado del sujeto y sus competidores para la totalidad de los sectores muestreados (Cuadro 4).

Considerando las variables del árbol sujeto, la correlación más alta se obtuvo entre el IDPA y el diámetro de copa (DC), que alcanzó un 0,72 . Le siguen en el diámetro a la altura del pecho (DAP) y el espacio ocupado por la copa (variable derivada).

\section{CUADRO 4}

CORRELACION ENTRE EL INCREMENTO DIAMETRAL MEDIO ANUAL (VARIABLE DEPENDIENTE) Y LAS VARIABLES DEL ARBOL SUJETO Y SUS COMPETIDORES

\begin{tabular}{|cc|}
\hline Variable del arbol sujeto & Coeficiente de correlacion \\
\hline DC & 0.716 \\
DAP & 0.548 \\
IC & 0.454 \\
ECD & 0.427 \\
t & 0.368 \\
DISTM & 0.321 \\
VT & 0.228 \\
HIC & 0.149 \\
\hline Variable de los arboles competidores & \\
\hline HICCO & 0.547 \\
HICO & 0.482 \\
DISTMCO & 0.345 \\
DAPMCO & 0.181 \\
DCMCO & 0.149 \\
\hline
\end{tabular}


Al considerar las variables de los árboles competidores, la mayor correlación, igual a 0,547 , se obtuvo con la altura de inicio de copa del competidor (HICCO). Le siguen la altura del árbol competidor (HTCO) y la distancia media entre los árboles competidores y el árbol sujeto (DISTMCO).

Con la finalidad de saber si las variables transformadas permitían obtener una correlación más alta con el incremento anual, se probaron una serie de transformaciones, desglosadas en el Cuadro 5. En términos generales se puede concluir que la transformación no mejoró las correlaciones. Sin embargo, se las debe considerar un antecedente importante para los modelos generales de crecimiento, donde inciden en el aumento de la correlación.

\section{CUADRO 5}

CORRELACION ENTRE EL CRECIMIENTO MEDIO DIAMETRAL ANUAL Y LAS VARIABLES TRANSFORMADAS PARA EL TOTAL DE LA MUESTRA ( $\mathrm{n}=75$ ARBOLES)

\begin{tabular}{cc}
\hline Variables transformadas & $\mathbf{r}$ \\
\hline DC $^{2}$ & 0.656 \\
DC x DAP & 0.594 \\
DAP x HT & 0.316 \\
1/E & 0.289 \\
1/DISTMCO & 0.333 \\
DAP 2 & 0.477 \\
Ln (HT) & 0.312 \\
HT/HTCO & 0.481 \\
ICO $_{2}$ & 0.210 \\
\hline
\end{tabular}

Construcción del modelo general para el crecimiento diametral anual. A partir del análisis de correlación simple entre el crecimiento en diámetro y las variables de los árboles sujeto y competidores, se seleccionaron algunas variables que en forma conjunta aumentaron la correlación. A través del análisis de regresión paso a paso se integraron dichas variables en modelos de aplicación práctica para la proyección del crecimiento de árboles individuales.

Se consiguieron tres modelos generales múltiples para predecir el incremento en diámetro. De acuerdo con el tipo de variable utilizada y su transformación, ellos pueden ser clasificados en: modelo general múltiple con o sin variables transformadas del árbol sujeto y modelo general múltiple con variables del árbol sujeto y de los competidores.

a) Modelo general múltiple con variables transformadas del árbol sujeto.

$$
\begin{aligned}
\mathrm{IDPA}= & -0.6381+0.0122 \mathrm{DC}^{2}+20.594 \frac{1}{\mathrm{E}}+0.1263 \mathrm{DAP}-0.0026 \mathrm{DAP}^{2} \\
& -0.3429 \ln (\mathrm{HT}) \\
\mathrm{r} \quad & 0.90 \\
\mathrm{ECMO}= & 24.7
\end{aligned}
$$


b) Modelo general múltiple con variables del árbol sujeto y distancia media al competidor.

$$
\begin{aligned}
\text { IDAPA } & =0.6949+0.0207 \text { DISTMCO }+0.0818 \mathrm{DC}-0.0306 \mathrm{E}+0.0297 \text { DAP } \\
\mathrm{r} & =0.89 \\
\mathrm{ECM} \% & =26.0
\end{aligned}
$$

c) Modelo general múltiple con variables del sujeto y de los competidores.

$$
\begin{aligned}
& \mathrm{IDPA}= 0.8273+0.0294 \text { DISTMCO }+0.0514 \mathrm{DC}-0.0274 \mathrm{E}+0.0301 \mathrm{DAP} \\
&-0.0465 \mathrm{HICCO}+0.0182 \mathrm{HIC} \\
& \mathrm{r} \quad=0.91 \\
& \mathrm{ECM} \%=23.7
\end{aligned}
$$

Las correlaciones de los tres modelos presentados son muy similares. Todos entregan un coeficiente de determinación superior al $80 \%\left(\mathrm{r}^{2}\right)$, de modo que pueden considerarse bastante confiables. Los modelos b y $\mathrm{c}$ tienen la ventaja sobre el modelo a de considerar indicadores directos de la competencia. Así, el modelo b incluye la distancia media al competidor y el modelo $\mathrm{c}$ incluye, además de ésta, la altura de inicio de copa del competidor.

Los tres modelos pueden utilizarse para estimar el crecimiento diametral aproximado del raulí joven. Como restricción en su uso deben tomarse en cuenta ciertos rangos de aplicación, que están limitados por la dispersión de la muestra para cada parámetro.

Los modelo b y c permiten estimar en forma aproximada el crecimiento individual del árbol para distintas clases de espaciamiento, de modo que hacen también posible determinar la reacción del individuo frente a intervenciones silvicolas que abren el rodal.

Análisis de sensibilidad del modelo. Para el análisis de sensibilidad se escogió el modelo general que incluye un mayor número de variables independientes (modelo c), con el objeto de probar distintas situaciones en las cuales se desarrolla la especie. Además, es el modelo que presenta el mayor coeficiente de correlación ( $\mathrm{r}$ ) y el menor error cuadrático medio (ECM\%).

$$
\begin{aligned}
\text { Modelo c IDAPA }= & 0.8273+0.0294 \text { DISTMCO }+0.0514 \mathrm{DC}-0.0274 \mathrm{E} \\
& +0.0302 \mathrm{DAP}-0.0465 \mathrm{HICCO}+0.0812 \mathrm{HIC}
\end{aligned}
$$

Para el análisis de sensibilidad la muestra se estratificó de acuerdo con la altura total (HT) de los árboles, variando en cada estrato la edad (E), la distancia media al competidor (DISTMCO), la altura de inicio de la copa del sujeto (HIC) y la altura de inicio de copa del competidor (HICCO). Los rangos de las variables consideradas en el análisis están en función del rango real encontrado para el estrato de la muestra. Los valores del diámetro a la altura del pecho (DAP) y del diámetro de copa de los árboles sujeto corresponden a valores promedios para el estrato de altura considerada.

En el cuadro 6 se entregan los valores del incremento anual en diámetro para distintas situaciones o combinaciones de variables de estado del árbol sujeto y sus competidores. La finalidad es proporcionar, sobre la base de supuestos cercanos a los valores reales, los incrementos en diámetro que pueden ser esperados para árboles de rauli que crecen en rodales mixtos.

Las tendencias que se obserwan en la variación del incremento en diámetro son las espera- 
das de acuerdo con la magnitud de los coeficientes de las variables de estado que participan en el modelo c).

Los valores de incremento en diámetro que aparecen en el Cuadro indicados con un asterisco son los máximos valores estimados, que fluctúan entre $1.01 \mathrm{~cm}$ y $1.26 \mathrm{~cm}$. Estos crecimientos ocurren en los tres estratos de altura total, en las clases de edad (de 14 a 25 años) y en la mínima altura de inicio de la copa del competidor (HICCO). Los mínimos valores de incre-

\section{CUADRO 6}

\section{RESULTADOS DEL ANALISIS DE SENSIBILIDAD PARA EL INCREMENTO DIAMETRAL ANUAL (EVALUACION DE LA FUNCION - IDAPA)}

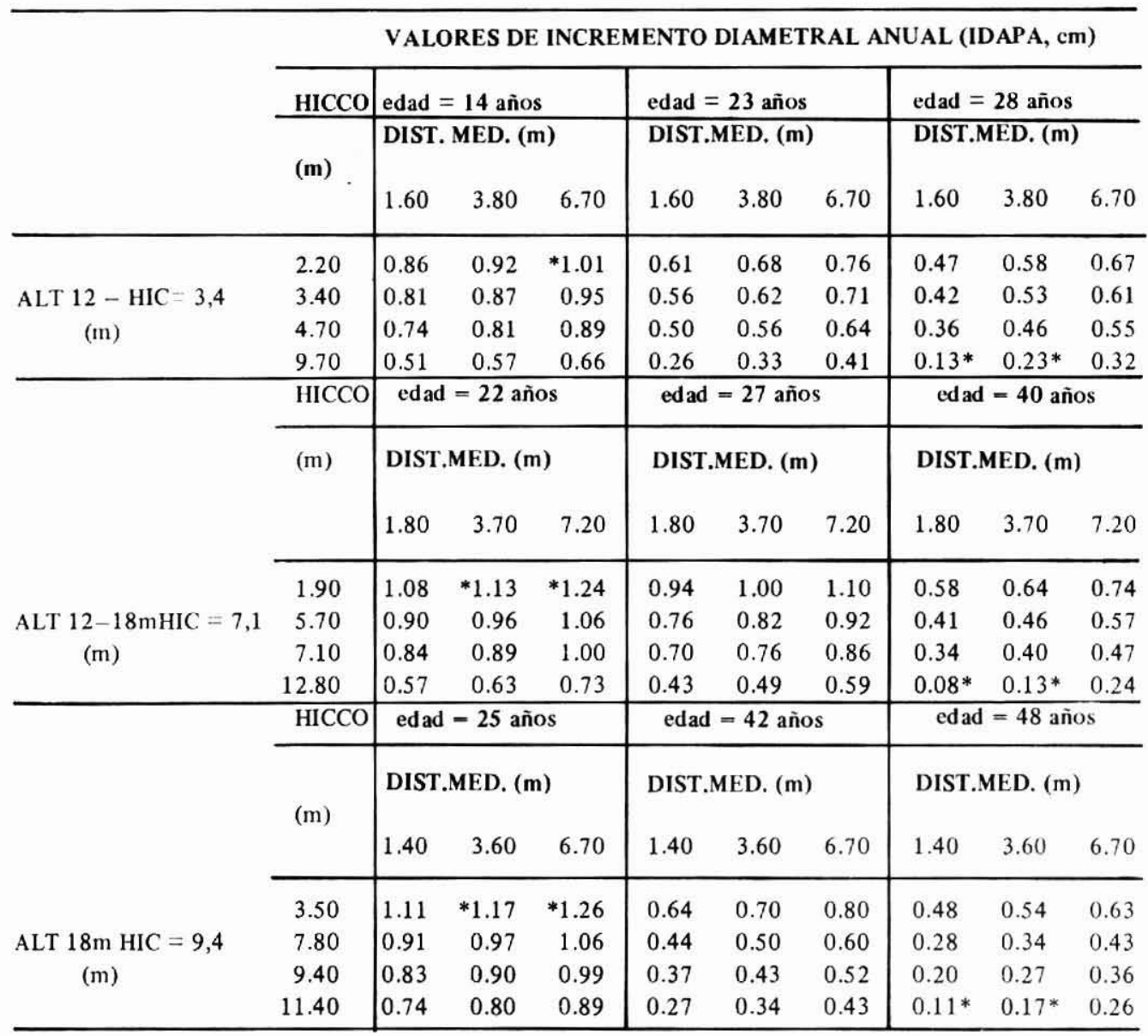

NOTA:

HICCO = altura de inicio de copa del competidor

HIC = altura de inicio de copa del árbol sujeto

DIST.MED. = distancia media al competidor. 
mento en diámetro, que fluctúan entre $0.08 \mathrm{~cm}$ y $0.23 \mathrm{~cm}$, ocurren en los tres estratos de altura total al coincidir la máxima altura de inicio de copa de los competidores (HICCO), la menor distancia entre el árbol sujeto y sus competidores (DISTMCO) y la clase de edad (entre 28 a 48 años).

\section{CONCLUSIONES}

El análisis de las muestras de raulí extraidas de rodales jóvenes en el sector de Panguipulli permiten, a pesar de concentrarse sólo en un área de su distribución, concluir lo siguiente:

El incremento diamétrico a la altura del pecho se puede estimar confiablemente considerando variables de estado corrientes del árbol, de sus competidores y la distancia entre ambos.

- Los tres modelos de crecimiento diametral desarrollados en base a una muestra de 75 árboles tienen un coeficiente de determinación superior al $80 \%$, razón por la cual se consideran bastante confiables.

Para el rango de edad de la muestra, que fluctúa entre 20 y 45 años, el incremento diametral promedio anual es de aproximadamente $0,7 \mathrm{~cm}$. Individuos que crecen espaciadamente superan $1 \mathrm{~cm}$ de crecimiento anual medio.

- Los antecedentes de crecimiento diametral que se pueden evaluar con los 3 modelos desarrollados constituyen una base para estimar rendimientos a nivel individual y del rodal. Los modelos permiten además conocer en forma aproximada cuál será la reacción de los árboles al modificar su situación de espaciamiento. En este sentido, pueden considerarse indicadores prácticos del crecimiento que registrarán los individuos al ser sometidos a intervenciones silvícolas tales como clareos y raleos.

\section{REFERENCIAS BIBLIOGRAFICAS}

ALVAREZ, S. y GROSSE, H. 1978. Antecedentes generales y análisis para el manejo de lenga (Nothofagus pumilio (Poepp. et Endl.) Endl) Krasser) en Alto Mañihuales, Aysén. Tesis, Universidad de Chile, Fac. de Cs. Forestales. Santiago, 144 p.

DONOSO, C. 1981. Tipos forestales de los bosques nativos de Chile. CONAF/PNUD/FAO, FO: DP/CHI/76/ 003. Santiago, Chile. Doc. de Trabajo No 38.70 p.

GROSSE, H.; CUBILLOS, V.A. y BOURKE, M. 1976. Investigación manejo silvícola de diferentes tipos de bosques nativos. Informe preliminar. Corporación de Fomento de la Producción/Instituto Forestal. Concepción, Chile.

HERRERA, J.D. y MAY, F.F. 1976. Caracterización y análisis para el ordenamiento de renovales de raulí (Nothofagus alpina Poepp. et Endl.) en Jaula, Provincia de Malleco. Tesis, Universidad de Chile, Fac. de Cs. Forestales. Santiago. 63 p.

KRAMER, H. und ACKA, A. 1982. Leitfaden für Dendrometrie and Bestandesinventur. J. D. Sauerländer's Verlag, Frankfurt A.M. 251 p.

PUENTE, M. et al. 1979. Manejo de renovales de raulí (Nothofagus alpina) y roble (Nothofagus obliqua). Identificación y caracterización de renovales de raulí y roble. CONAF/PNUD/FAO, FO: DP/CHI/ 76/003. Santiago, Chile. Doc. de Trabajo No 29.88 p., anexos.

SOLER, M.P. 1979. Análisis evolutivo y comportamiento de renovales no intervenidos de raulí (Nothofagus alpina Poepp. et Endl.) según edad. Tesis, Universidad de Chile, Fac. de Cs. Forestales. Santiago. 59 p. 